\title{
Human miR-31 targets radixin and inhibits migration and invasion of glioma cells
}

\author{
DASONG HUA ${ }^{1}$, DONG DING ${ }^{1}$, XU HAN $^{1}$, WEIYI ZHANG ${ }^{1}$, NA ZHAO $^{1}$, \\ GREGORY FOLTZ ${ }^{2}$, QING LAN ${ }^{4}$, QIANG HUANG ${ }^{4}$ and BIAOYANG LIN ${ }^{1-3}$ \\ ${ }^{1}$ Division of Systems Biology, Zhejiang - California International Nanosystems Institute (ZCNI), Zhejiang \\ University, Hangzhou 310029, P.R. China; ${ }^{2}$ Swedish Neuroscience Institute, Swedish Medical Center, \\ Seattle, WA 98122; ${ }^{3}$ Department of Urology, University of Washington, Seattle, WA 98195, USA; \\ ${ }^{4}$ Department of Neurosurgery and Brain Tumor Research Laboratory, Second Affiliated \\ Hospital of Soochow University, Suzhou 215004, P.R. China
}

Received August 14, 2011; Accepted October 20, 2011

DOI: 10.3892/or.2011.1555

\begin{abstract}
MicroRNAs (miRNAs) are a novel group of short RNAs, about 20-22 nucleotide in length, that regulate gene expression in a post-transcriptional manner by affecting the stability or translation of mRNAs and play important roles in many biological processes. Many microRNAs have been implicated in glioblastoma. miR-31 is dysregulated in several types of cancer including colon, breast, prostate, gastric and lung cancers. However, the expression and role of miR-31 in glioblastoma are still unclear. In this study, we performed real-time reverse transcriptase polymerase chain reaction (RT-PCR) assays on 10 glioblastoma and 7 normal brain tissues. We found that miR-31 is down-regulated in glioblastoma compared with normal brain tissues. Ectopic expression of miR-31 inhibited migration and invasion ability of U251 glioma cells. Expression profiling analysis revealed that miR-31 affected the cell migration and motility process by regulating migration and invasion related genes. Finally, we demonstrated that miR-31 targeted radixin predominantly via inhibition of protein translation instead of degradation of mRNA.
\end{abstract}

Correspondence to: Dr Biaoyang Lin, Division of Systems Biology, Zhejiang - California International Nanosystems Institute (ZCNI) Zhejiang University, 268 Kaixuan Road, Hangzhou, Zhejiang 310029, P.R. China

E-mail: biaoylin@gmail.com

Abbreviations: U251-Control, U251 cell transduced with pMIRNA1control viral particles; U251-miR-31, U251 cell transduced with pMIRNA1-miR-31 viral particles; RDX, radixin; miRNA, microRNA; pmirGLO-RDX-WT, pmirGLO vector contains 3'-UTR of RDX; pmirGLO-RDX-MUT, pmirGLO-RDX-WT mutated in the region of predicted miR-31 binding site

Key words: glioma, miR-31, radixin, invasion

\section{Introduction}

The annual incidence of malignant glioma is approximately 5 cases per 100,000 people [Central Brain Tumor Registry of the Unites States (CBTRUS) 2009 statistical report, www.cbtrus. org]. Despite aggressive surgery, radiation and chemotherapy, the median survival is only 12-15 months for glioblastoma (GBM) (1). Better understanding of the mechanism and finding new therapeutic targets for glioma is urgent. miRNAs are a novel group of short RNAs, about 20-22 nucleotide in length, that regulate gene expression in a post-transcriptional manner by affecting the stability or translation of mRNAs (2). Many microRNAs have been implicated in glioblastoma $(3,4)$. Ciafr et al examined the global expression levels of 245 microRNAs in glioblastoma multiforme by microarray and identified a group of miRNAs including a strongly up-regulated expression of miR-221 and down-regulated expression of a set of brain-enriched miRNAs including miR-128, miR181a, miR-181b and miR-181c in glioblastoma (5).

miR-31 expression was down-regulated in human carcinomas of the breast (6), prostate (7), ovary (8) and stomach (9), but paradoxically up-regulated in human colorectal (10), liver (11), head-and-neck tumors (12) and squamous cell carcinomas of the tongue (13). It can either elicit promoting or inhibitive effects on cancers depending on the organs where the cancers arise. In breast cancer, miR-31 inhibits metastasis by targeting ITGAR, RDX and RhoA (6); but in lung cancer, miR-31 acts as an oncogenic miRNA by repressing tumor suppressor LATS2 and PPP2R2A (14). In colon carcinoma cells, miR-31 expression is regulated by TGF- $\beta$ and it targets TIAM1 to regulate migration and invasion (15). However, the role of miR-31 in GBM has not been studied, and which of the targets identified in other cancer types are used by GBM cells remains unknown.

In this study, we confirmed underexpression of miR-31 in glioblastoma samples compared with normal brain tissues. In addition, we showed that ectopic expression of miR-31 inhibits migration and invasion ability of U251 glioma cells. A microarray analysis revealed that miR-31 could regulate many 


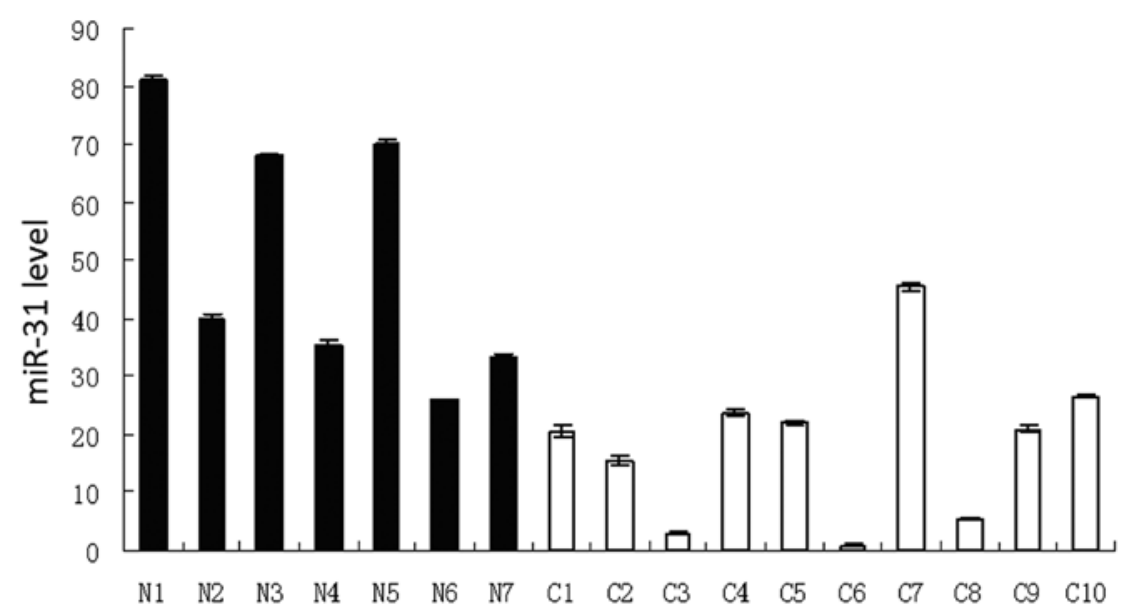

Figure 1. miR-31 was down-regulated in glioma tissues compared to normal brain tissues (Student's t-test, P<0.01). Real-time PCR analysis of miR-31 in 7 normal brain tissues (N, black columns) and 10 glioma tissues (C, white columns), RNA input was normalized by human U6 snRNA.

migration and invasion related genes. Finally, we demonstrated that miR-31 targeted radixin in GBM cells to regulate migration and invasion processes.

\section{Materials and methods}

Sample information and RNA isolation. Tissue samples were obtained after informed consent from adult patients diagnosed with glioblastoma de novo or other neurological diseases with IRB approval. They were freshly resected during surgery and immediately frozen in liquid nitrogen for subsequent total RNA extraction. RNA was isolated from tissues using mirVana ${ }^{\mathrm{TM}}$ miRNA Isolation Kit (Ambion, Austin, TX) according to the manufacturer's instruction.

miRNA stem-loop RT-PCR. Expression of miR-31 was quantified using stem-loop RT-PCR analysis and carried out in triplicate for each sample. The relative amount of miRNAs was normalized against U6 snRNA, and the fold change for each miRNA was calculated by the $2-\Delta \Delta \mathrm{Ct}$ method (16). The primers used for stem-loop RT-PCR are available upon request.

Cell culture. U251 was purchased from Cell Bank of Chinese Academy of Sciences (Shanghai) and maintained in high glucose DMEM supplemented with $10 \%$ fetal bovine serum (FBS) and penicillin/streptomycin $(100 \mu \mathrm{g} / \mathrm{ml})$.

Virus package and transduction. pMIRNA1-control vector was purchased from SBI (System Biosciences, USA). Hsa-miR-31 precursor with about $100 \mathrm{bp}$ flanking sequences on both sides was cloned from the human genome. The PCR primers are available upon request. The sequence was inserted into pMIRNA1 at the EcoRI and NotI sites to construct pMIRNA1miR-31. The construct was validated by sequencing. Virus packaging and U251 transduction were performed according to the manufacturer's protocol (SBI).

Wound healing assay. Cells $\left(1 \times 10^{6}\right)$ were seeded on 6-well plates. An artificial homogeneous wound was created on the monolayer cells with a sterile plastic $100 \mu 1$ micropipette tip on the second day. After wounding, the debris was removed by washing the cells with serum-free medium and the cells were grown in normal medium. Photographs of the wound area were taken using a Nikon Eclipse TS100 microscope (under $\mathrm{x} 100)$ at 0 and $48 \mathrm{~h}$.

Transwell assay and Transwell matrix penetration assay. Cells $\left(1 \times 10^{4}\right)$ in $200 \mu \mathrm{l}$ serum-free DMEM were plated on the upper compartment of a Transwell device (Corning, USA). For the Transwell matrix penetration assay, the Transwell filter was coated with Matrigel (BD Biosciences). The lower compartment was filled with $600 \mu \mathrm{l}$ of DMEM complete medium. After $48 \mathrm{~h}$, the cells inside the upper chamber were removed with cotton swabs, and the cells on the lower membrane surface were fixed in methanol and stained with $0.1 \%$ crystal violet. Photographs were taken using a Nikon Eclipse TS100 microscope (under $\mathrm{x} 100$ ). Six visual fields of each filter were randomly selected and counted under a microscope. All experiments were performed in triplicate.

GeneChip mRNA array and Gene Ontology analysis. Affymetrix U133 plus 2 GeneChip (Affymetrix, Santa Clara, CA, USA) was used to identify genes regulated by miR-31. The CEL files were imported into the Affymetrix's Expression Console (V1.1) program and data normalized using RMA normalization. Data were then exported to the Genespring Program (Agilent, Inc.) was for further analysis. To filter out those lowly expressed genes across the chips, probes with intensities $<100$ in both chips were excluded from the analysis. Genes changed $>2$-fold were considered as significant. Gene Ontology (GO) analysis was performed using the online HighThroughput GoMiner program (17).

Plasmids construction and luciferase activities analysis. 3'UTR of radixin containing predicted binding site of miR-31 was cloned by PCR and inserted into the EcoRI and XhoI sites of pmirGLO vector (Promega, Inc.), which is designed to quantitatively evaluate miRNA activity by introducing the miRNA target sites (or their mutated counterparts) down- 


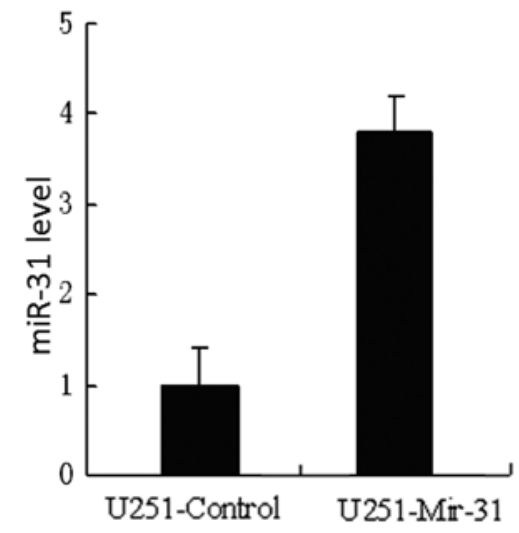

Figure 2. Real-time PCR analysis of miR-31 in U251 cells after transduction with miR-31 (U251-miR-31) or negative control (U251-Control). RNA input was normalized by human U6 snRNA.

stream of the firefly luciferase gene (luc2). Mutations in the binding site region were generated by the MutanBest Kit (Takara, Inc.) according to manufacturer's instructions. Both pmirGLO-RDX-WT and pmirGLO-RDX-MUT constructs were validated by sequencing (Shanghai Sangon Biological Engineering Technology and Services Co., Ltd.). Forty-eight hours after transfection, luciferase activities were measured in infinite M200 (TECAN) using Luciferase Assay System (Promega, Inc.) according to the manufacturer's protocol.

Western blot analysis. Western blot analysis was performed by the standard protocol. The radixin monoclonal antibody was from Epitomics, Inc. Anti-GAPDH antibody was from MultiSciences Biotech Co., Ltd., China).

Statistical analyses and data access. Data are presented as mean \pm SEM. Student's t-test was used for comparisons and
$\mathrm{P}<0.05$ was considered significant. All supplementary data are available at http://systemsbiozju.org/data/Dasong_Huang/ Supplementary/.

\section{Results}

miR-31 is down-regulated in human glioblastoma tissues compared with normal brain tissues. We confirmed a previous observation that miR-31 is underexpressed in GBM (18) using 10 glioblastoma tissues and 7 normal brain tissues. The results showed that miR-31 is down-regulated significantly in glioblastoma compared with normal brain tissues (Student's t-test, $\mathrm{P}<0.01$; Fig. 1).

miR-31 inhibits migration and invasion of glioblastoma cells. To study the function of miR-31 in glioma, we made a pMIRNA1-miR-31 to transduce U251 glioblastoma cells. After transduction, the overexpression of miR-31 in U251-miR-31 compared with U251-Control was confirmed by RT-PCR to be 3.78 -fold (Fig. 2). We then tested the polarized migration of cells using the wound healing assay. We found that the scratchwound closure speed of U251-miR-31 was lower than that of U251-Control (Fig. 3). In addition, a transwell invasion assay showed that miR-31 could inhibit migration and invasion of U251 in vitro (Fig. 4). However, an MTT assay showed miR-31 did not have effect on U251 proliferation (data not shown).

Expression profiling revealed that miR-31 is mostly involved in migration and invasion pathways in GBM. To understand the global effect of miR-31 on glioblastoma cells, we performed microarray analysis using Affymetrix U133 plus2 GeneChip to compare gene expression profile of U251-miR-31 to that of U251-Control cells. The data were deposited to the NCBI Gene Expression Omnibus and are accessible through GEO Series accession no. GSE28810 (http://www.ncbi.nlm.nih.gov/ geo/query/acc.cgi?acc=GSE28810). Using a 2-fold change as a
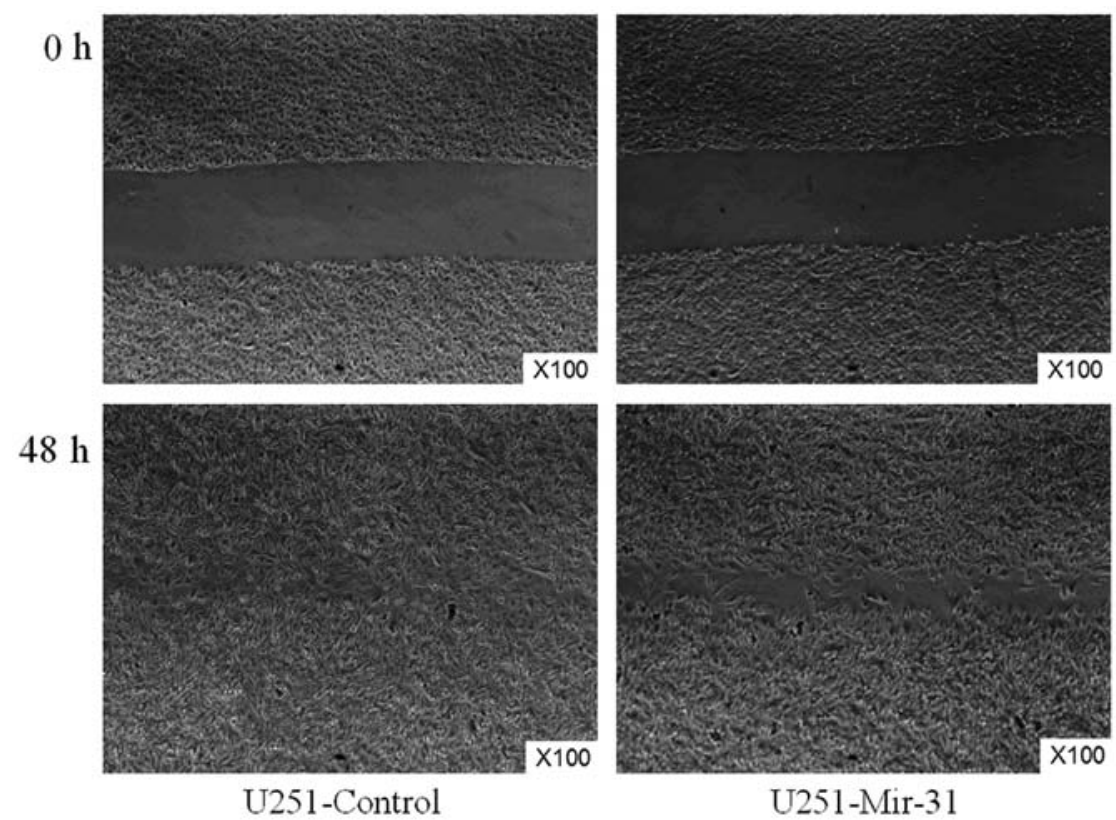

Figure 3. miR-31 inhibited wound closure speed of U251. Magnification, x100. 
A

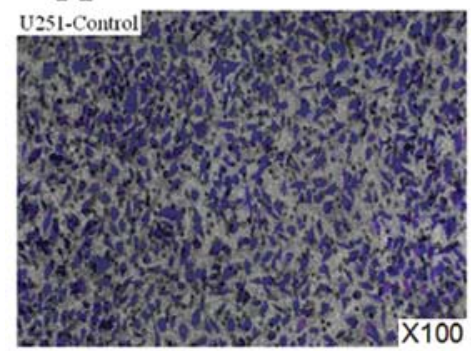

B

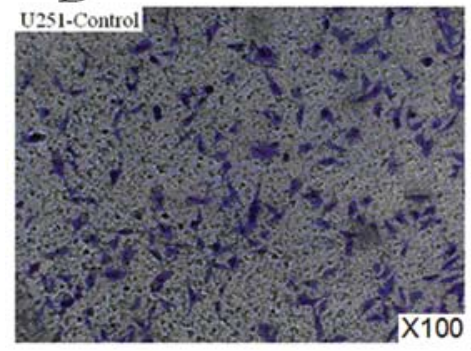

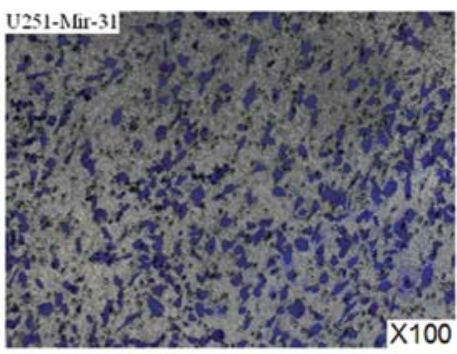
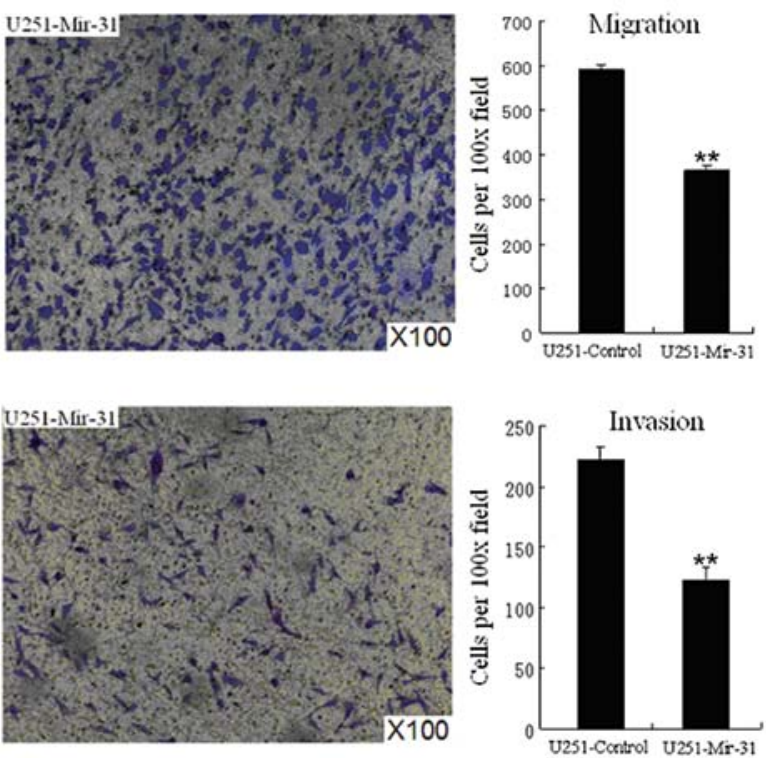

Figure 4. miR-31 negatively regulated glioma cell migration and invasion in vitro. (A) The migratory U251-miR-31 and U251-Control cells were stained and counted under microscope. The migratory cell number of U251-miR-31 transfected cells was significantly less than that of U251-Control cells (346.17 \pm 25.21 vs. $586.83 \pm 14.16)$. (B) The invasive U251-miR-31 and U251-Control cells were stained and counted under microscope. The invasive cell number of U251-miR-31 transfected cells was significantly less than that of U251-Control cells (122.66 \pm 8.32 vs. 222.66 \pm 9.07$)$. Magnification, $\mathrm{x} 100{ }^{* *} \mathrm{P}<0.01$.

A

hsa-mir-31 5'AGGCAAG-AUGCUGGCAUAGCU-3'

IIIIII RDX3UTR 3'ACCGUUCCACCCUACGUAAGGU-5 (2158-2137]

hsa-mir-31 5'-AGGCAAG-AUGCUGGCAUAGCU-3

IIII

RDX 3UTR(MUT) 3-AVAUGCUCACCCUACGUAAGGU.5 21258-2137

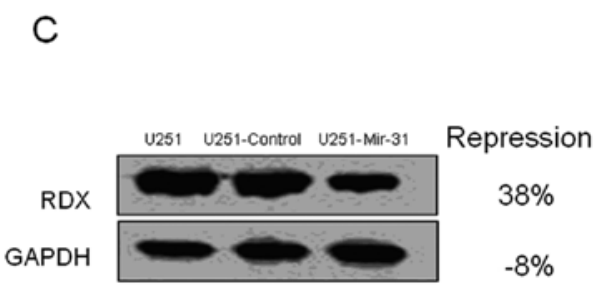

B

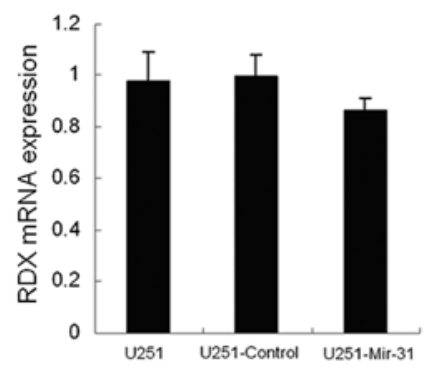

D

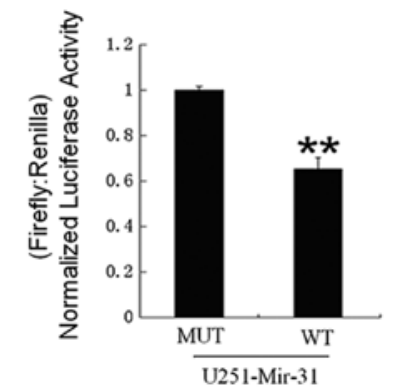

Figure 5. Radixin expression is inhibited by miR-31 in U251 cells. (A) RDX has a predicted binding site for miR-31 in its 3 'UTR. The sequences in the boxed region were mutated to generate pmirGLO-RDX-WT vector. (B) The expression of RDX in mRNA level was measured by RT-PCR. (C) RDX protein expression is reduced by $46 \%$ after transducting miR-31 to U251 cells as determined by Western blotting. (D) Relative luminescence of U251-miR-31 transfection of pmirGLO-RDX-WT or pmirGLO-RDX-MUT vectors. ${ }^{*} \mathrm{P}<0.05,{ }^{* *} \mathrm{P}<0.01$.

cut-off value, we identified 347 (115 down-regulated and 232 up-regulated) genes that were differentially expressed (data available upon request). Five of the differential expressed genes were randomly selected and their expression changes were confirmed by RT-PCR (data not shown).

The differentially expressed genes were then grouped by Gene Ontology analysis using GoMiner using GO terms at level 3 for biological processes, with all genes detected in the microarray as the total input. Twenty-five GO terms were found to be enriched, including angiogenesis (GO:0001525), cell motility (GO:0048870) regulation of locomotion (GO:0040012) and cell migration (GO:0016477) (Table I). Fifty-five genes were identified in the GO terms related to migration and invasion, consistent with our observation that miR-31 was involved in the migration and invasion of glioma cells.

miR-31 directly targets the radixin gene. In an attempt to understand how miR-31 regulate migration and invasion and 


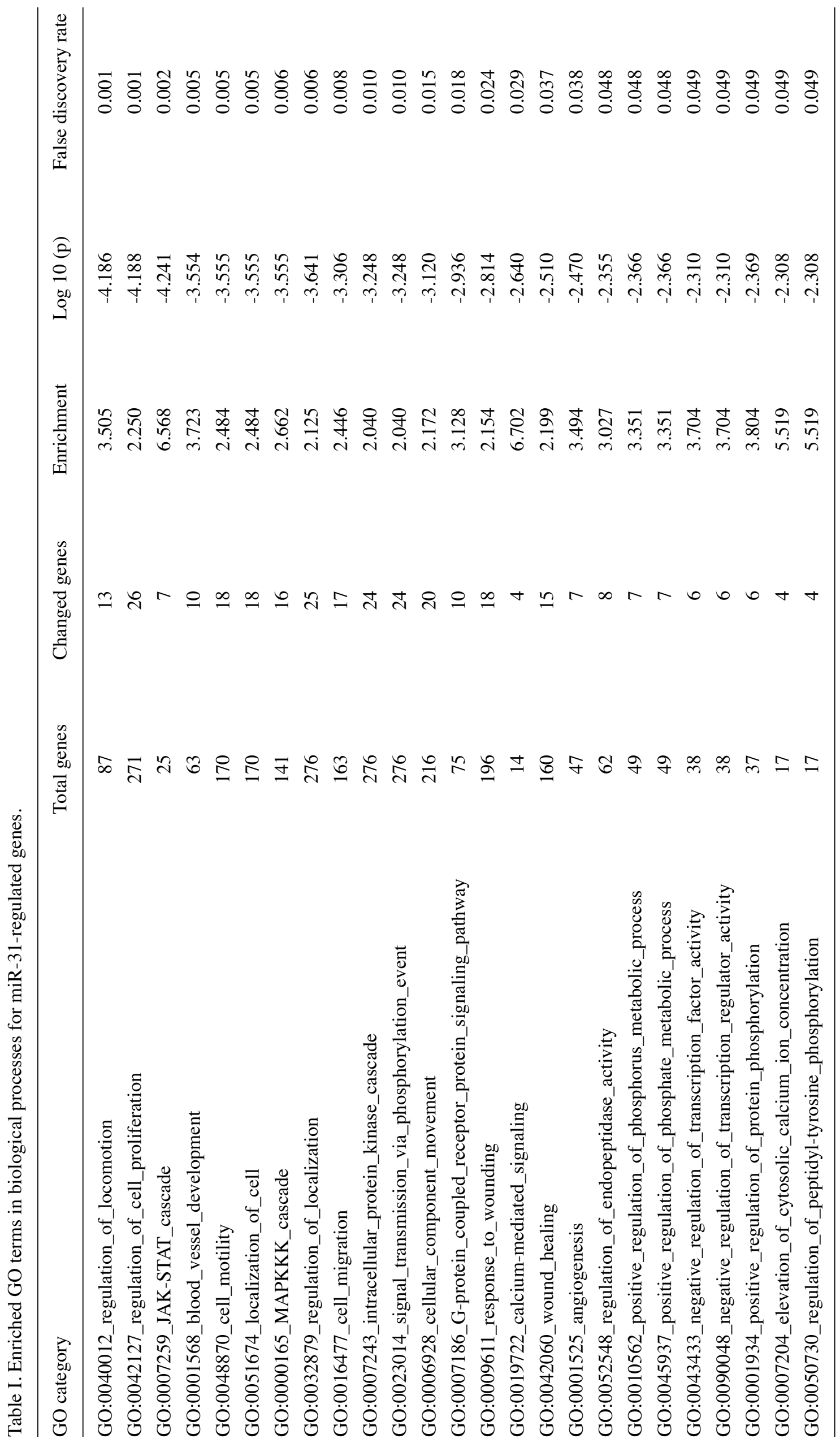


to identify which target genes are involved in the process, we predicted the targets of miR-31 using three commonly used bioinformatics tools (TargetScan, PicTar and miRanda) (19-21). All of the three software predicted that radixin (RDX) is a potential targeted gene of miR-31 (Fig. 5A). Radixin is a cytoskeletal protein and was shown to be a target of miR-31 in breast cancer $(6,22)$. We hypothesized that miR-31 might target radixin in glioblastoma as well. To test this hypothesis, we measured the expression of radixin in U251-miR-31 and U251-Control at both mRNA and protein levels. The results showed that miR-31 inhibits RDX at protein level (Fig. 5C), but not at mRNA level (Fig. 5B).

To further confirm that RDX is a target gene for miR-31, 3'UTR of RDX containing the predicted binding site was amplified from human genomic DNAs and subsequently inserted into pmirGLO to construct pmirGLO-RDX-WT. pmirGLO is designed to quantitatively evaluate miRNA activities by engineering miRNA target sites (or mutated target sites) downstream of the firefly luciferase gene (luc2). pmirGLO-RDX-MUT (with mutated target sites) was constructed by mutating the seed region (boxed) in pmirGLO-RDX-WT (Fig. 5A). Transfection of pmirGLORDX-WT into U251-miR-31 cells led to $35 \%$ reduction of luciferase activity compared to that of the cells transfected with pmirGLO-RDX-MUT (Fig. 5D), further confirming that miR-31 indeed targets the predicted seed regions in the 3'UTR of the RDX gene.

\section{Discussion}

MiRNA are a class of small non-coding RNAs that participate in various biological processes by regulating mRNA stability or translation. A great number of miRNAs have been found to act as either oncogenes or tumor suppressors in glioma. For instance, miR-146b can inhibit glioma cell migration and invasion by targeting MMPs (23). miR-21 is a well known oncogenic miRNA that acts as an antiapoptotic factor and promotes glioma proliferation $(24,25)$. miR-10b is overexpressed in malignant glioma and associated with proteins involved in tumor invasion such as UPAR and RhoC (26). Kefas et al identified down-regulation of miR-7 in glioblastomas and found that miR-7 directly targets EGFR, and affected the epidermal growth factor receptor (EGFR) and the Akt pathways in glioblastoma cells (27).

miR-31 is abnormally expressed in several types of cancers and plays a crucial role in tumor metastasis (28). In this study, we demonstrated that miR-31 is down-regulated in glioblastoma compared to normal tissues, and migration and invasion of U251 cells in vitro were inhibited by miR-31. We further demonstrated that that radixin is a direct target of miR-31 in glioma. Valastyan et al showed that miR-31 repressed the expression of radixin (6) and that expression of radixin alone could partially phenocopy the effects of miR-31 on metastasis in breast cancer (29). This indicated that miR-31 may play similar roles in GBM as in breast cancer.

Expression profiing showed that ectopic expression of miR-31 in U251 cells significantly affect the expression of many migration and invasion related genes, which include TGFBR1, EMP1 and RGS4. EMP1and RGS4 were recently validated as the direct target of miR-31 in oesphageal squa- mous cell carcinoma (30). However, whether EMP1 and RGS4 are direct targets of miR-31 in glioma cells remains to be studied.

In conclusion, our data showed that miR-31 plays important roles in glioma migration and invasion and miR-31 or its target gene radixin could be used as potential targets for the development of therapeutic approaches for inhibiting migration and invasion of glioma cells.

\section{Acknowledgements}

This study was supported by grant 81072060 from the National Natural Science Foundation of China.

\section{References}

1. Wen PY and Kesari S: Malignant gliomas in adults. N Engl J Med 359: 492-507, 2008.

2. Bartel DP: MicroRNAs: cenomics, biogenesis, mechanism, and function. Cell 116: 281-297, 2004.

3. Delfino KR, Serao NV, Southey BR and Rodriguez-Zas SL: Therapy-, gender- and race-specific microRNA markers, target genes and networks related to glioblastoma recurrence and survival. Cancer Genomics Proteomics 8: 173-183, 2011.

4. Hummel R, Maurer J and Haier J: MicroRNAs in brain tumors: a new diagnostic and therapeutic perspective? Mol Neurobiol 44: $1-12,2011$.

5. Ciafr SA, Galardi S, Mangiola A, et al: Extensive modulation of a set of microRNAs in primary glioblastoma. Biochem Biophys Res Commun 334: 1351-1358, 2005.

6. Valastyan S, Reinhardt F, Benaich N, et al: A pleiotropically acting microRNA, miR-31, inhibits breast cancer metastasis. Cell 137: 1032-1046, 2009.

7. Schaefer A, Jung M, Mollenkopf HJ, et al: Diagnostic and prognostic implications of microRNA profiling in prostate carcinoma. Int J Cancer 126: 1166-1176, 2009.

8. Creighton CJ, Fountain MD, Yu Z, et al: Molecular profiling uncovers a p53-associated role for microRNA-31 in inhibiting the proliferation of serous ovarian carcinomas and other cancers. Cancer Res 70: 1906-1915, 2010.

9. Guo J, Miao Y, Xiao B, et al: Differential expression of microRNA species in human gastric cancer versus non-tumorous tissues. J Gastroenterol Hepatol 24: 652-657, 2009.

10. Motoyama K, Inoue H, Takatsuno Y, et al: Over- and underexpressed microRNAs in human colorectal cancer. Int J Oncol 34: 1069-1075, 2009.

11. Wong QW, Lung RW, Law PT, et al: MicroRNA-223 is commonly repressed in hepatocellular carcinoma and potentiates expression of Stathmin1. Gastroenterology 135: 257-269, 2008.

12. Liu CJ, Tsai MM, Hung PS, et al: miR-31 ablates expression of the HIF regulatory factor FIH to activate the HIF pathway in head and neck carcinoma. Cancer Res 70: 1635-1644, 2010.

13. Wong TS, Liu XB, Wong BY, Ng RW, Yuen AP and Wei WI: Mature miR-184 as potential oncogenic microRNA of squamous cell carcinoma of tongue. Clin Cancer Res 14: 2588-2592, 2008.

14. Liu X, Sempere LF, Ouyang H, et al: MicroRNA-31 functions as an oncogenic microRNA in mouse and human lung cancer cells by repressing specific tumor suppressors. J Clin Invest 120 : 1298-1309, 2010

15. Cottonham CL, Kaneko S and Xu L: miR-21 and miR-31 converge on TIAM1 to regulate migration and invasion of colon carcinoma cells. J Biol Chem 285: 35293-35302, 2010.

16. Chen C, Ridzon DA, Broomer AJ, et al: Real-time quantification of microRNAs by stem-loop RT-PCR. Nucleic Acids Res 33: e179, 2005.

17. Zeeberg B, Feng W, Wang G, et al: GoMiner: a resource for biological interpretation of genomic and proteomic data. Genome Biol 4: R28, 2003.

18. Silber J, Lim DA, Petritsch C, et al: miR-124 and miR-137 inhibit proliferation of glioblastoma multiforme cells and induce differentiation of brain tumor stem cells. BMC Med 6: $14,2008$.

19. Krek A, Grun D, Poy MN, et al: Combinatorial microRNA target predictions. Nat Genet 37: 495-500, 2005. 
20. Lewis BP, Burge CB and Bartel DP: Conserved seed pairing, often flanked by adenosines, indicates that thousands of human genes are microRNA targets. Cell 120: 15-20, 2005.

21. John B, Enright AJ, Aravin A, Tuschl T, Sander C and Marks DS: Human MicroRNA targets. PLoS Biol 2: e363, 2004.

22. Hoeflich KP and Ikura M: Radixin: cytoskeletal adopter and signaling protein. Int J Biochem Cell Biol 36: 2131-2136, 2004.

23. Xia H, Qi Y, Ng SS, et al: microRNA-146b inhibits glioma cell migration and invasion by targeting MMPs. Brain Res 1269 158-165, 2009.

24. Chen Y, Liu W, Chao T, et al: MicroRNA-21 down-regulates the expression of tumor suppressor PDCD4 in human glioblastoma cell T98G. Cancer Lett 272: 197-205, 2008.

25. Chan JA, Krichevsky AM and Kosik KS: MicroRNA-21 is an antiapoptotic factor in human glioblastoma cells. Cancer Res 65: 6029-6033, 2005.

26. Sun L, Yan W, Wang Y, et al: MicroRNA-10b induces glioma cell invasion by modulating MMP-14 and uPAR expression via HOXD10. Brain Res 1389: 9-18, 2011.
27. Kefas B, Godlewski J, Comeau L, et al: microRNA-7 inhibits the epidermal growth factor receptor and the Akt pathway and is down-regulated in glioblastoma. Cancer Res 68: 3566-3572, 2008.

28. Valastyan S and Weinberg RA: miR-31: A crucial overseer of tumor metastasis and other emerging roles. Cell Cycle 9: 2124-2129, 2010.

29. Valastyan S, Chang A, Benaich N, Reinhardt F and Weinberg RA: Concurrent suppression of integrin alpha5, radixin, and RhoA phenocopies the effects of miR-31 on metastasis. Cancer Res 70: 5147-5154, 2010.

30. Zhang T, Wang Q, Zhao D, et al: The oncogenetic role of microRNA-31 as a potential biomarker in esophageal squamous cell carcinoma. Clin Sci (Lond) 121: 437-447, 2011. 